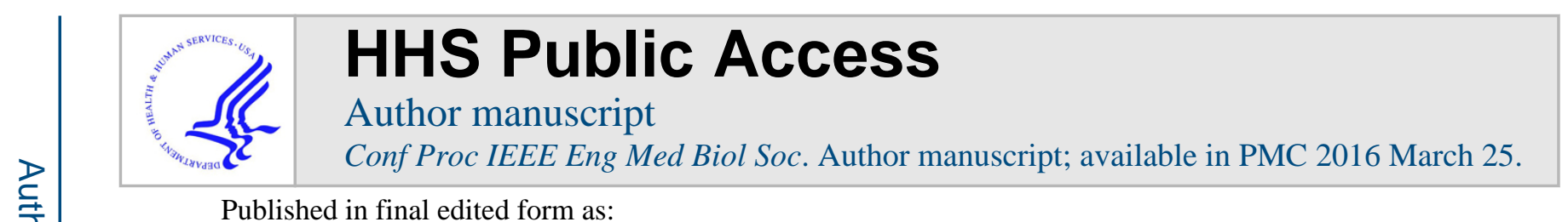

Published in final edited form as:

Conf Proc IEEE Eng Med Biol Soc. 2011 ; 2011: 761-764. doi:10.1109/IEMBS.2011.6090174.

\title{
Millimeter-Scale Epileptiform Spike Patterns and Their Relationship to Seizures
}

\author{
Ann C. Chamberlain, \\ Jonathan Viventi, \\ Justin A. Blanco, \\ Dae-Hyeong Kim, \\ John A. Rogers, and \\ Brian Litt [Senior Member IEEE] \\ Ann C. Chamberlain: achamb@seas.upenn.edu; Jonathan Viventi: jviventi@seas.upenn.edu; Justin A. Blanco: \\ blancoj@seas.upenn.edu; Dae-Hyeong Kim: dkim98@illinois.edu; John A. Rogers: jrogers@uiuc.edu; Brian Litt: \\ littb@mail.med.upenn.edu
}

\section{Abstract}

Advances in neural electrode technology are enabling brain recordings with increasingly fine spatial and temporal resolution. We explore spatio-temporal (ST) patterns of local field potential spikes using a new high-density active electrode array with $500 \mu \mathrm{m}$ resolution. We record subdural micro-electrocorticographic $(\mu \mathrm{ECoG})$ signals in vivo from a feline model of acute neocortical epileptiform spikes and seizures induced with local administration of the GABA antagonist, picrotoxin. We employ a clustering algorithm to separate 2-dimensional (2-D) spike patterns to isolate distinct classes of spikes unique to the interictal and ictal states. Our findings indicate that the 2-D patterns can be used to distinguish seizures from non-seizure state. We find two statistically significant ST patterns that uniquely characterize ictal epochs. We conclude that millimeter-scale ST spike dynamics contain useful information about ictal state. This finding may be important to understanding mechanisms underlying local circuit activity during seizure generation. Further work will investigate whether patterns we identify can increase our understanding of seizure dynamics and their underlying mechanisms and inform new electrical stimulation protocols for seizure termination.

\section{I. introduction}

In epilepsy research, many different brain recording techniques have been employed to understand neural dynamics between, prior to and during seizures. Electrophysiologic studies employ techniques that range in size and scale from the Utah array [1], which records multi-unit activity from penetrating electrodes $400 \mu \mathrm{m}$ apart, to scalp Electroencephalography (EEG), which records electrical potentials that are distorted by the skull, scalp, cerebrospinal fluid (CSF) and soft tissues, from electrodes many centimeters apart. Other studies utilize modalities ranging from Magnetoencephalography (MEG) and functional Magnetic Resonance Imaging (fMRI) to Voltage Sensitive Dyes (VSDs). MEG is a non-invasive technique that records magnetic components of potentials perpendicular to 
the EEG, and suffers from low spatial resolution, similar to EEG. Image-based technologies such as fMRI and VSDs complement standard electrophysiology, though each has its own spatial and temporal resolution limitations. In this study, we use a new, high-spatial density subdural surface active electrode array of 360 channels covering an area of $10 \mathrm{~mm} \times 9 \mathrm{~mm}$ to measure local field potential (LFP)-scale electrical signals in vivo from an acute feline epilepsy model. We test the hypothesis that there are spatio-temporal (ST) patterns of LFP spike activity recorded on a millimeter-scale that are unique to seizures.

\section{Methods}

\section{A. Animal Recording}

We analyzed micro-electrocorticographic ( $\mu \mathrm{ECoG})$ data from an acute in vivo feline model of epilepsy. Adult cats were anesthetized with a continuous infusion $(3 \sim 10 \mathrm{mg} / \mathrm{kg} / \mathrm{hr})$ of intravenous thiopental. A craniotomy and durotomy were performed to expose a $2 \times 3 \mathrm{~cm}$ region of cortex. The high resolution electrode array was then placed on the surface of the brain over primary visual cortex, localized by electrophysiological recordings of visual evoked potentials. Picrotoxin, a GABA-A receptor antagonist that blocks inhibition, was topically applied adjacent to the anterior-medial corner of the electrode array in an amount sufficient to induce abnormal electrical spikes and seizures from the covered region [2].

The active electrode array placed on the cortex was used to record data from 360 independent channels arranged in 20 columns and 18 rows, spaced $500 \mu \mathrm{m}$ apart. Each electrode contact was composed of a $300 \mu \mathrm{m} \times 300 \mu \mathrm{m}$ square of platinum. Two highperformance, flexible silicon transistors for each electrode buffered and multiplexed the recorded signals[3]. The total array size was $10 \mathrm{~mm} \times 9 \mathrm{~mm}$. Signals were recorded with an effective sampling rate of $277.7 \mathrm{~Hz}$ per channel. Figure 1 is a photograph of the array placed on the surface of the visual cortex of a cat.

We analyzed 13 minutes and 40 seconds of data for this study, containing 724 automatically detected spikes and 2 seizures, verified by expert review.

\section{B. Pre-processing}

All recordings were first band pass filtered between 1 and $50 \mathrm{~Hz}$ using a 6th-order butterworth filter in the forward and reverse direction, using Matlab's filtfilt function, to achieve zero-phase filtering.

64 of 360 channels were non-functional due to manufacturing imperfections. The missing data for these channels were interpolated from the surrounding electrodes using a 2-D averaging spatial filter of window size $3 \times 3$ pixels.

Small offsets in time that result from row multiplexing were corrected by upsampling and shifting the data in order to accurately align rows in time. Data were first upsampled by the number of rows within the array (18) via Matlab's interp function, which applies a low pass filter interpolation algorithm. Data were then temporally shifted by their respective row offset. 


\section{Spike Detection}

We used a voltage-threshold detector to detect spikes on the signal obtained by averaging all 360 channels. The voltage threshold was set by visual inspection at $-500 \mu \mathrm{V}$. When the average signal crossed the threshold from above, a $160 \mathrm{msec}$ segment of the recording was stored (60 msec prior to the crossing and $100 \mathrm{msec}$ post-crossing).

We analyzed only single spikes (i.e. no poly-spikes) and retained only spikes which occurred on a majority of the electrodes in the 2-D array in order to simplify the analysis of spike propagation. Specifically, the following quantitative criteria were used to retain spikes: 1) a single negative-going threshold crossing followed by a single positive-going threshold crossing within the clipped $160 \mathrm{msec}$ window surrounding the triggering threshold crossing; 2) at least $50 \%$ of electrodes have a root-mean-square (RMS) value $>40 \%$ of the maximal RMS across channels (within the $160 \mathrm{msec}$ window). 724 average spikes met the above criteria. Figure 2 shows 16 representative detected spike waveforms.

\section{Feature Selection}

For each of the 724 spikes, a 63 -element feature vector was generated in the following manner: The average spike waveform was cross-correlated with each of the 360 singlechannel spikes. This yielded a single value per channel capturing the delay of the spike on each channel of the array. The zero-meaned RMS (i.e. standard deviation) for each channel was then calculated. This yielded a single value per channel capturing a representation of the power of the spike on each channel of the array. The $724 \times 360$ delay values were then normalized by dividing by each row maximum. The $724 \times 360$ 'power' values were similarly normalized. The $724 \times 360$ matrix of delay values was then concatenated with the $724 \times 360$ matrix of 'power' values. The result was a $724 \times 720$ matrix representing 724 spikes, each with 720 features. Principal components analysis (PCA) was performed and a number of dimensions accounting for $>99 \%$ of the data variance was retained. This resulted in a dimensionality reduction from 720 to 63 .

\section{E. Clustering}

k-mediods clustering [4] was performed and the gap statistic [5] was used to determine the number of clusters, similar to methods applied in other EEG classification tasks [6]. 16 clusters of ST patterns were identified. Figure 3 shows delay maps for spikes clustered closest in L1 distance to the centroid of each distinct cluster. Blue indicates electrodes with early delay values relative to the average spike waveform and red indicates electrodes with later delay values. All analysis was performed in the Matlab environment (The Mathworks Inc., Natick, MA, U.S.A.)

\section{F. Statistical Testing}

We hypothesized that some ST patterns would occur preferentially during seizure epochs. We used Pearson's chi-squared test to test the null hypothesis that the proportion of spikes occurring during seizure is equal across clusters (i.e. ST patterns). To address the issue of identifying a specific cluster which might account for a rejection of the null hypothesis, we conducted a permutation test. We held cluster membership of each spike fixed while randomly permuting the seizure and non-seizure labels for one million permutations. For 
every permutation we recorded the maximum (over all clusters) of the proportion of seizurespikes within each cluster to obtain the null distribution. We then compared the observed maximum to this null distribution.

\section{Results}

Figure 4 displays spike clustering and seizure analysis results. Each of the pies represents one of the 16 different spike ST patterns identified. Delay maps of representative spikes from each corresponding cluster are shown in Figure 3. The whole area of each pie in Figure 4 is scaled in proportion to the total number of spikes within the cluster. The blue section within each pie represents the percentage of spikes associated with non-seizure epochs. The red section within each pie represents the percentage of spikes recorded during seizures. Clusters 3 and 6 appear to have disproportionately large numbers of spikes occurring during seizure relative to outside.

We found a strong relationship between ST pattern and seizure state (i.e. within or outside of a seizure epoch). We reject the null hypothesis that the proportion of spikes occurring during seizure in each of the 16 clusters was the same $\left(\chi^{2}(15, \mathrm{~N}=724)=415.1, \mathrm{p} \ll 0.0001\right)$. Furthermore, we found the proportion of within-seizure spikes contained specifically in clusters 3 and 6 were significantly higher than would be expected by chance $(\mathrm{p} \ll 0.0001$ for both clusters).

\section{Discussion}

Our initial analysis indicates that two specific ST spike patterns correlate with seizure epochs. In addition, we found other ST spike patterns that appear to be more loosely associated with seizures. We speculate that those patterns indicate periods of transition from the interictal to ictal states. We believe these patterns may hold information about the progression of abnormal electrical activity as seizures approach. We propose that analyzing the brain's electrical activity using this novel electrode array will provide new opportunities to increase our understanding of epileptiform spikes and their patterns of propagation. One possibility raised by these waveforms, is that spikes and seizures, when viewed at this resolution, may have features in common with cardiac dysrhythmias. In this sense it may be precisely their multidimensional ST appearance that could reveal re-entrant patterns and triggers in the same way that these types of events occur in cardiac tissue. We next plan to study the relationship among waveforms leading into ictal events, those occurring periodically during seizures, and the waveforms that occur immediately prior to seizure cessation.

Recordings at this spatial scale may be important to clinical patient care and evaluation for epilepsy surgery, as evidenced by studies in humans of high frequency oscillations and microseizures that are poorly detected by standard clinical electrode systems[7,8]. We anticipate that this new electrode technology, combined with novel methods for analyzing the large, high-resolution data sets arising from it, may lead to better understanding of spike discharges and seizure development, and more effective therapies for the more than $33 \%$ of epilepsy patients who remain medically refractory. 


\section{Acknowledgments}

This material is based upon work supported by the National Science Foundation under grant DMI-0328162 and the U.S. Department of Energy, Division of Materials Sciences under Award No. DE-FG02-07ER46471, through the Materials Research Laboratory and Center for Microanalysis of Materials (DE-FG02-07ER46453) at the University of Illinois at Urbana-Champaign. J.A.R. acknowledges a National Security Science and Engineering Faculty Fellowship. This work was also supported by the National Institutes of Health Grants (NINDS RO1-NS041811, NINDS R01 NS 48598) and the Dr. Michel and Mrs. Anna Mirowski Discovery Fund for Epilepsy Research. J.V. was supported by the National Institutes of Health under Ruth L. Kirschstein National Research Service Award 2T32HL007954 from the NIH-NHLBI.

\section{References}

1. Campbell PK, Jones KE, Huber RJ, Horch KW, Normann Ra. A silicon-based, three-dimensional neural interface: manufacturing processes for an intracortical electrode array. IEEE transactions on bio-medical engineering. Aug.1991 38:758-68. [PubMed: 1937509]

2. Morimoto K, Fahnestock M, Racine RJ. Kindling and status epilepticus models of epilepsy: rewiring the brain. Progress in neurobiology. May.2004 73:1-60. [PubMed: 15193778]

3. Viventi J, Kim DH, Moss JD, Kim YS, Blanco Ja, Annetta N, Hicks a, Xiao J, Huang Y, Callans DJ, Rogers Ja, Litt B. A Conformal, Bio-Interfaced Class of Silicon Electronics for Mapping Cardiac Electrophysiology. Science Translational Medicine. 2010; 2:24ra22-24ra22.

4. Hastie, T.; Tibshirani, R.; Friedman, J. The Elements of Statistical Learning. New York, New York, USA: Springer-Verlag; 2001.

5. Tibshirani R, Walther G, Hastie T. Estimating the number of clusters in a data set via the gap statistic. Journal of the Royal Statistical Society: Series B (Statistical Methodology). May.2001 63:411-423.

6. Blanco JA, Stead M, Krieger A, Viventi J, Marsh WR, Lee KH, Worrell Ga, Litt B. Unsupervised classification of high-frequency oscillations in human neocortical epilepsy and control patients. Journal of neurophysiology. Nov.2010 104:2900-12. [PubMed: 20810694]

7. Worrell, Ga; Gardner, AB.; Stead, SM.; Hu, S.; Goerss, S.; Cascino, GJ.; Meyer, FB.; Marsh, R.; Litt, B. High-frequency oscillations in human temporal lobe: simultaneous microwire and clinical macroelectrode recordings. Brain : a journal of neurology. 2008; 131:928-37. [PubMed: 18263625]

8. Stead M, Bower M, Brinkmann BH, Lee K, Marsh WR, Meyer FB, Litt B, Van Gompel J, Worrell $\mathrm{Ga}$. Microseizures and the spatiotemporal scales of human partial epilepsy. Brain : a journal of neurology. Oct.2010 133:2789-97. [PubMed: 20685804] 


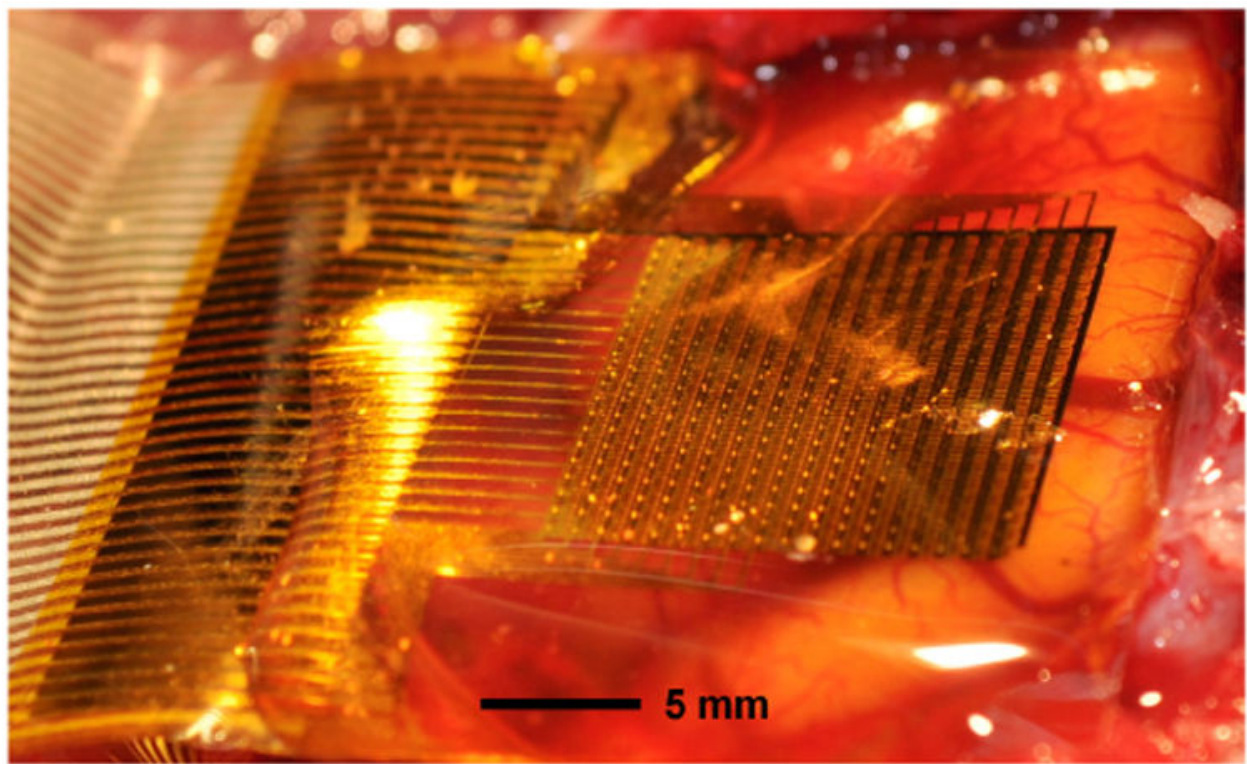

Fig. 1.

Photograph of a 360 channel, high density neural electrode array used in a feline model of epilepsy. The electrode array is placed on the surface of visual cortex. The electrode size and spacing is $300 \mu \mathrm{m} \times 300 \mu \mathrm{m}$ and $500 \mu \mathrm{m}$, respectively. 


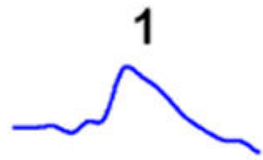

5

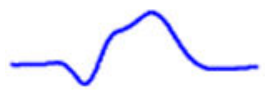

9

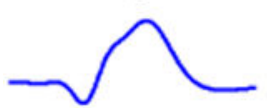

13

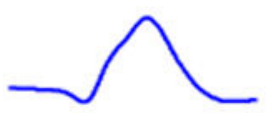

2

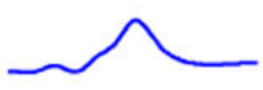

6

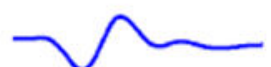

10

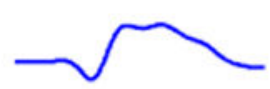

14

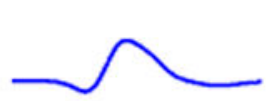

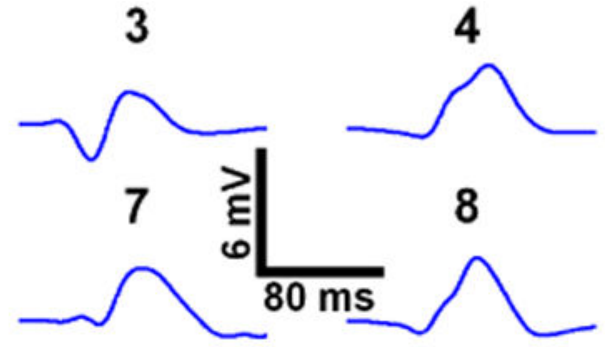

12

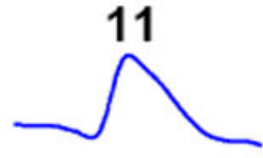

15

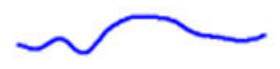

16

Fig. 2.

Representative spike waveforms retained by the spike detection algorithm. Each of the spike waveforms is $160 \mathrm{~ms}$ clipped from the average voltage recording of all 360 channels. Within each window there is only one negative-going crossing followed by one positive-going crossing. These 16 waveforms are of the spikes closest (in the L1 sense) to each of the cluster centroids and correspond to the spatio-temporal delay maps in Figure 3. Negative is plotted up by convention. 
1

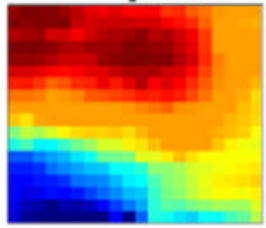

5

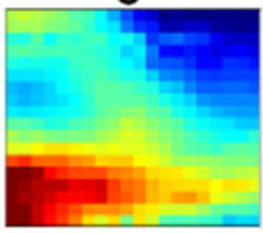

9

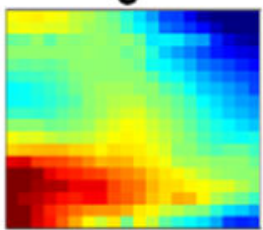

13

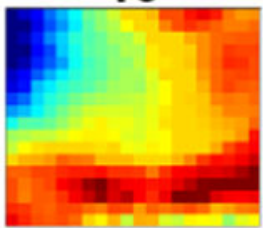

2

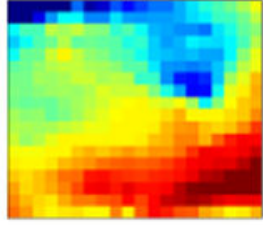

6

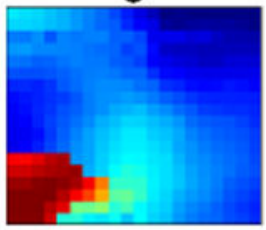

10

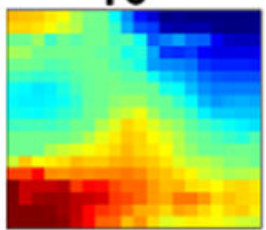

14

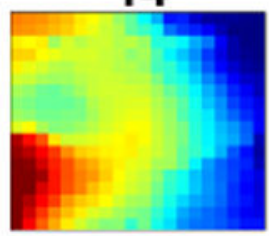

3

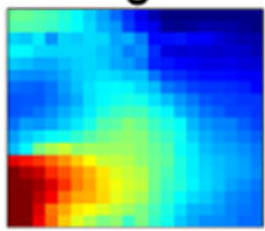

7

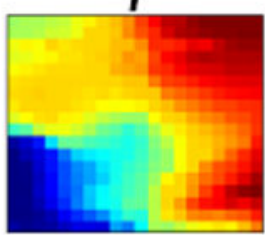

11

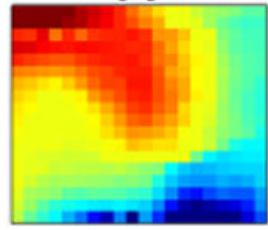

15

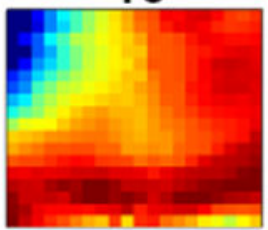

4

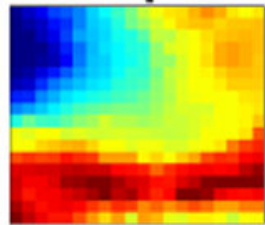

8

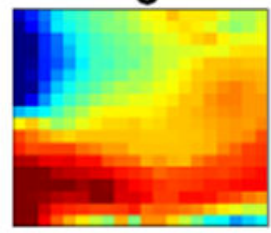

12

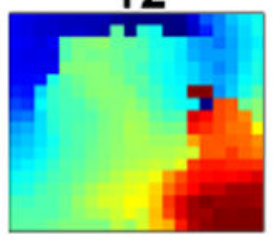

16

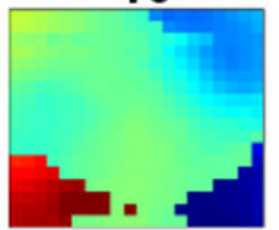

Fig. 3.

Delay maps for the 16 clusters. Each of the above delay maps represents the spike within each cluster that is closest to its cluster centroid. Blue indicates electrodes with an early delay value relative to the average spike waveform and red indicates electrodes with a late delay value. Color shading represents relative timing of peak voltage in each spike, but not speed of propagation. As an example, the delay map for cluster 1 displays a spike ST pattern of propagation across the array of a spike that enters on the bottom left and proceeds in a sweeping arc until it exits the array in the top left. The corresponding RMS maps have not been included due to space constraints. 


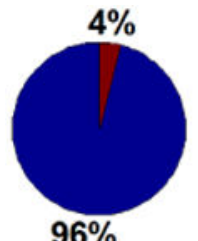

$96 \%$
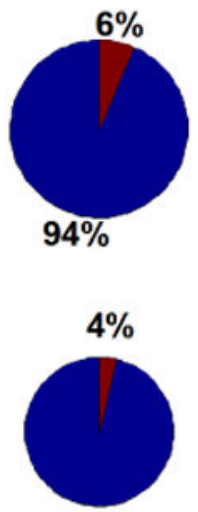

$96 \%$

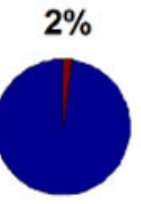

$98 \%$

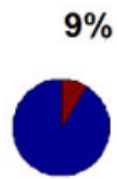

$91 \%$

$50 \%$

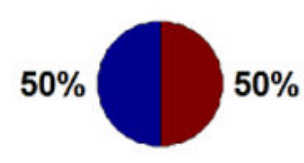

9\%

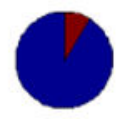

$91 \%$

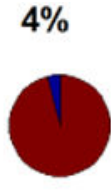

$96 \%$
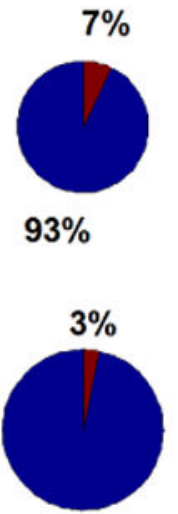

$97 \%$

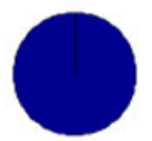

$100 \%$
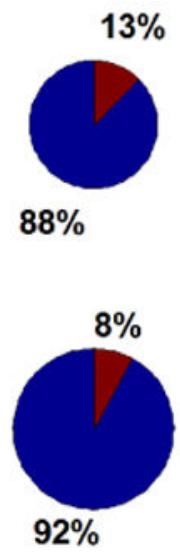

$2 \%$

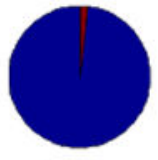

$98 \%$

$13 \%$

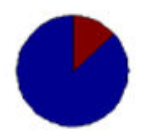

$87 \%$

\section{Non-seizure}

Seizure

Fig. 4.

Pie charts representing the 16 identified clusters and their proportion of spikes during and outside of seizures. Each of the pie charts represents one of the 16 different spike ST patterns identified. The whole area of each pie is scaled in proportion to the total number of spikes within the cluster. The blue section within each pie is the percentage of spikes associated with non-seizure epochs. The red section within each pie is the percentage of spikes recorded during seizures. 